[RADIOCARbon, Vol 25, No. 1, 1983, P 39-58]

\title{
BRITISH MUSEUM NATURAL RADIOCARBON MEASUREMENTS XVI
}

\author{
RICHARD BURLEIGH, JANET AMBERS, \\ and KEITH MATTHEWS
}

Research Laboratory, The British Museum, London WC1B 3DG, England

The following list consists of dates for archaeologic and geologic samples mostly measured from June 1981 to June 1982. The dates were obtained by liquid scintillation counting of benzene using the laboratory procedures outlined in previous lists (see, eg, BM-VIII, R, 1976, v 18, $\mathrm{p}$ 16). Dates are expressed in radiocarbon years relative to AD 1950 based on the Libby half-life for ${ }^{14} \mathrm{C}$ of $5570 \mathrm{yr}$, and are corrected for isotopic fractionation $\left(\delta^{13} \mathrm{C}\right.$ values are relative to $\left.\mathrm{PDB}\right)$. No corrections have been made for natural ${ }^{14} \mathrm{C}$ variations (although in some instances approximate calibrated dates taken from the tables of R M Clark (1975) have been given in the comments where this aids interpretation of results). The modern reference standard is NBS oxalic acid (SRM 4990). Errors quoted with dates are based on counting statistics alone and are equivalent to \pm 1 standard deviation $( \pm 1 \sigma)$. Descriptions, comments, and references to publications are based on information supplied by submitters.

\section{ARCHAEOLOGIC SAMPLES}

\section{Cherchel series}

$$
\text { Algeria }
$$

Samples from pit containing successive kilns in Roman Forum at Cherchel $\left(36^{\circ} 40^{\prime} \mathrm{N}, 1^{\circ} 40^{\prime} \mathrm{W}\right)$. Coll 1979 and subm by $\mathrm{T}$ Potter, Dept Prehist and Romano-British Antiquities, British Mus.

\section{BM-1909. Cherchel}

Charcoal.

BM-1910. Cherchel

Charcoal, ref C79, 159.

$$
1760 \pm 130
$$$$
\delta^{13} C=-25.4 \% \text { o }
$$

$$
1620 \pm 70
$$

$\delta^{13} \mathrm{C}=-23.0 \%$

General Comment (TP): on archaeol grounds kilns have terminus post quem of ca $\mathrm{AD} 450$, but lie in deep pit within ruins of church, built ca AD 420, with substantial timber elements. Wood burned in kilns may have been seasoned timber from structure of church. Full pub will be in Benseddik, N and Potter, T W, Excavations at the Forum site at Cherchel: Archéol Algér Bull (supp vol), ms in preparation.

\section{British Isles}

Late Pleistocene/early Holocene mammalian extinctions and related samples

Bone samples (collagen fractions) from larger terrestrial mammals now extinct in Britain, dated as part of study of latest dates of survival 
of these sp in British Isles (R, 1976, v 18, p 30-31; R, 1982, v 24, p 262-269; Clutton-Brock and Burleigh, in press and in preparation).

\section{BM-1725. Kildale}

$$
\begin{array}{r}
\mathbf{8 2 7 0} \pm \mathbf{8 0} \\
\delta^{1.3} C=-19.3 \% o
\end{array}
$$

1lth dorsal vertebra of bull (Bos primigenius) from skeleton found in peaty marl deposit between Kildale Hall and R Leven, Kildale Park, N E Yorkshire, England (54 $25^{\prime} \mathrm{N}, 1^{\circ} 5^{\prime} \mathrm{W}$, Natl Grid Ref NZ 609097). Coll 1968 by R Close and R H Hayes and subm 1980 by Caroline Grigson, Odontol Mus, Royal Coll Surgeons, London. Dated for comparison with age of peaty marl (Zone IV/V interface by pollen analysis) and date for peat directly surrounding part of skeleton, 10,350 \pm 200 : GaK-2707, unpub (Jones, 1971, p 84-86, 90; Simmons, 1975, p 57). Comment (CG): result shows skeleton dates to Pollen Zone VI (late Boreal/Mesolithic period) and is thus among earlier group of dates for remains of Bos primigenius in Britain ( $c f$ BM-1841: $8620 \pm 80, \mathrm{R}, 1982$, v 24, p 264), but does not agree with dating based on pollen evidence and GaK-2707 implying earliest Bos primigenius known from Britain (Grigson, 1978, p 50-53).

\section{BM-1807. Thor's Fissure}

$20,100 \pm 1900$

Calcaneum of reincer (Rangifer turanchus) from Buxton $=-19.3 \%$

langifer tarandus) from Buxton Mus colln, labeled as from Thor's Fissure, Manifold Valley, Staffordshire, England $\left(53^{\circ} 5^{\prime} \mathrm{N}, 1^{\circ} 55^{\prime} \mathrm{W}\right.$, Natl Grid Ref SK 090540). Coll 1950 and subm 1980 by D Bramwell, Peakland Archaeol Soc and R Jacobi, Dept Classics and Archaeol, Univ Lancaster. Comment (RJ): result suggests presence of reindeer in S Peak Dist at time of max spread of Devensian ice-sheet.

\section{BM-1889. Corhampton}

$4790 \pm 70$

Cranial (occipital) bone of Bos primigenius found in assoc with Windmill Hill style pottery during drainage excavation at Corhampton, Hampshire, England ( $51^{\circ} 0^{\prime} \mathrm{N}, 1^{\circ} 10^{\prime} \mathrm{W}$, Natl Grid Ref SU 609202). Coll 1951 by F Cottrill and subm 1981 by C Grigson to provide date for Bos remains and assoc pottery. Comment (CG): result agrees with archaeol evidence and pattern of clates for other remains of Bos primigenius in $S$ Britain (Grigson, in press).

\section{BM-1980. Gugh}

$$
\text { Modern }
$$

Thoracic vertebra of juvenile $B$ os from articulated partial sketeo found at $2 \mathrm{~m}$ depth below estimated original surface in partial skeleton Pleistocene periglacial slope deposit ("Ram" or "Head") just above rocky shore, midway between Cow and Calf Rock and Kittern Rock, N end of Gugh I, Is of Scilly, Cornwall, England $\left(49^{\circ} 55^{\prime} \mathrm{N}, 6^{\circ} 20^{\prime} \mathrm{W}\right.$, Natl Grid Ref SV 890080). Coll 1981 by B Tucker and subm by A J Sutcliffe, Dept Palaeontol, British Mus (Nat Hist). Comment (RB): large size of bones (juvenile with unfused epiphyses; growth incomplete) suggested remains of Bos primigenius and hence possible transport (as live animal 
o: meat) to Is of Scilly by prehistoric man in Neolithic or Bronze age period, but date shows remains are those of recent large ox, possibly derived from shipwreck.

\section{Netherton series}

Samples from deserted late Saxon and Medieval manor site at Netherton, Faccombe, Hampshire, England $\left(51^{\circ} 20^{\prime} \mathrm{N}, 1^{\circ} 25^{\prime} \mathrm{W}\right.$, Natl Grid Ref SU 374575). Coll 1977 to 1979 and subm by J Fairbrother, Dept Archaeol, Univ Southampton (Webster, 1978; 1979; 1980).

BM-1899. Netherton $920 \pm 35$

Charcoal, ref NE/77 N10 $2189 \mathrm{~B} 1$, from industrial area.

BM-1900. Netherton

$1000 \pm 100$ industrial Pit 2643.

BM-1901. Netherton

$$
1000 \pm 80
$$

Charcoal, ref NE/78 P9 2648B, from late Saxon industrial Pit 2648.

$\mathbf{7 2 0} \pm \mathbf{5 0}$

BM-1902. Netherton $\delta^{13} \mathrm{C}=-25.9 \%$

Combined charcoal samples, ref 9 Q4 3358 and NE/79 P5 3331A, from post holes of late Saxon aisled hall, Bldg 2.

\section{BM-2006. Netherton}

$710 \pm 80$

Charcoal, ref NE/79 O4 3354, from late Saxon aisled hall, Bldg 2. Measured as check on BM-1902, above.

\section{BM-1905. Maldon}

$$
\begin{array}{r}
\mathbf{1 5 0} \pm \mathbf{5 0} \\
\delta^{13} \mathrm{C}=-19.6 \%
\end{array}
$$

Collagen from limb bones (radius, femur, and tibia) of domestic pig from complete skeleton found at ca $\mathrm{lm}$ depth during excavation of late Iron age and early Roman salt-works at Osea Road Red Hill, Maldon, Essex, England ( $51^{\circ} 45^{\prime} \mathrm{N}, 0^{\circ} 45^{\prime} \mathrm{E}$, Natl Grid Ref TL 887075). Coll 1972 by Kay de Brisay, Colchester Archaeol Group and subm 1981 by S Payne, Cambridge (de Brisay, 1972; 1973). Skeleton could not be dated from stratigraphic evidence (date for charcoal from site, Q-1173: $2130 \pm 40, \mathrm{R}, 1975, \mathrm{v} 17, \mathrm{p} 48$ ). Comment (SP): skeleton (Payne, 1981) is evidently relatively recent and not contemporary with Iron age salt extraction workings.

\section{South Lodge Camp series}

Samples from Deverel-Rimbury enclosed settlement site at South Lodge Camp, Berwick St John, Cranborne Chase, Wiltshire, England $\left(50^{\circ} 50^{\prime} \mathrm{N}, 2^{\circ} 0^{\prime} \mathrm{W}\right.$, Natl Grid Ref ST 954174), assoc with characteristic pottery and metalwork and close to Deverel-Rimbury barrow cemetery. 
Coll 1980 during re-excavation of site by J Barrett and R Bradley (Barrett et al, in press) and subm by $\mathrm{R}$ Bradley, Univ Reading. Comment by $\mathrm{R}$ Bradley.

\section{BM-1917 South Lodge Camp}

$2790 \pm 70$

Charcoal, ref BPA 005, assoc with cremated bone in fill of Bucket Urn, buried in deposit of flint nodules in top of ditch fill of Barrow 3.

\section{BM-1918. South Lodge Camp}

$2680 \pm 110$

a ? tion within group of burials outside Barrow 3.

\section{BM-1919. South Lodge Camp}

$2910 \pm 60$

$\delta^{13} \mathrm{C}=-25.0 \%$

Charcoal, ref BPA 011, from unurned cremation assoc with burned flint outside Barrow 3.

\section{BM-1920. South Lodge Camp}

$$
\begin{array}{r}
\mathbf{2 6 6 0} \pm \mathbf{6 0} \\
\delta^{13} C=-26.4 \% \text { o }
\end{array}
$$

Charcoal, ref BPA 023/01, assoc with cremation and fragmented bronze spearhead (Taunton industrial phase) cut into top of ditch fill of Barrow 2.

\section{BM-1921. South Lodge Camp}

$3020 \pm 60$

Charcoal, ref SL80 F529a, from fill of post hole of principal round house within enclosed settlement. Enclosure is aligned on bldg and sample should therefore date use of enclosure.

\section{BM-1922. South Lodge Camp}

$\mathbf{2 8 9 0} \pm \mathbf{5 0}$

Charcoal, ref SL80 F529b, from same context as BM-1921, above.

\section{BM-2023. South Lodge Camp}

$\mathbf{2 6 8 0} \pm \mathbf{5 0}$

Charcoal, ref BPA 80 023/01, from same context as BM-1920, above.

\section{BM-2024. South Lodge Camp}

$$
2730 \pm 70
$$

Charcoal, ref BPA $80014 \mathrm{~B}$, assoc with cremation outside Barrow 3 and overlain by base of globular urn.

General Comment (RB): BM-1921, -1922 are consistent with traditional date for site, suggested by metalwork and Taunton industrial phase, but enclosure is secondary to earlier field system that respects several nearby barrows. Remaining dates are for secondary burials in Barrows 2 and 3 of this group, the primary burials having been excavated by General Pitt Rivers in late 19th century. BM-1920, -1921 suggest Barrow 2 may be of later date than Barrow 3. Overall dates are entirely consistent with those from Handley Barrow 24 (BM-1644 to -1649, R, 1981, v 23, p 20-21), and similar enclosure at Down Farm (BM-1850 to -1854, R, 1982, 
v 24, p 271) and show continued use of Deverel-Rimbury pottery during late Bronze age in this area. Dates also suggest longer currency for Taunton metalwork than previously supposed.

\section{BM-1923. Poundbury}

$1500 \pm 40$

Collagen from human femur and tibia from inhumation ref PC80/ 1425, decapitation burial in Iron age ditch on edge of late 5 th century AD Romano-British cemetery at Poundbury Camp, Dorchester, Dorset, England $\left(50^{\circ} 40^{\prime} \mathrm{N}, 2^{\circ} 25^{\prime} \mathrm{W}\right.$, Natl Grid Ref SY 685912). Coll 1980 by C Green and A Graham and subm by Theya Molleson, British Mus (Nat Hist), to provide date for possible survival of pagan practice (Green, 1974; Harman, Molleson and Price, 1981). Comment (RB): result provides late date for decapitation burial in Britain within Christian era.

\section{Street House Farm series}

Samples from barrow site at Street House Farm, Loftus, Cleveland, England $\left(54^{\circ} 30^{\prime} \mathrm{N}, 0^{\circ} 50^{\prime} \mathrm{W}\right.$, Natl Grid Ref NZ 736196). Coll 1980 and subm by B E Vyner, Cleveland Co Council, Archaeol Sec.

BM-1966. Street House Farm

Charcoal, ref 30, from Plank 3, Quad 26.

BM-1967. Street House Farm

$$
4720 \pm 60
$$$$
\delta^{13} C=-26.3 \%
$$$$
4620 \pm 60
$$$$
\delta^{13} \mathrm{C}=-25.1 \%
$$

Charcoal, ref 56 , from vertical post 9 , id as Quercus sp by Rowena Gale, Royal Botanic Gardens, Kew.

BM-1968. Street House Farm

$$
4690 \pm 60
$$

Charcoal, ref 33, from Plank 3, Quad 22.

$\delta^{1.3} C=-25.9 \%$

\section{BM-1969. Street House Farm}

$\mathbf{4 7 2 0} \pm \mathbf{5 0}$

$\delta^{13} \mathrm{C}=-26.1 \%$

Charcoal, ref 57, from vertical post 10 , id as Quercus sp by $\mathrm{R}$ Gale.

\section{BM-2007. Street House Farm}

$$
\begin{array}{r}
\mathbf{3 2 2 0} \pm \mathbf{4 5} \\
\delta^{13} C=-24.9 \%
\end{array}
$$

Charcoal, ref 85 , id as Quercus sp by $\mathrm{R}$ Gale, from interface between Bronze age cairn and Neolithic mound.

\section{BM-2008. Street House Farm}

$$
\begin{array}{r}
2485 \pm 45 \\
\delta^{1 s} C=-24.4 \% o
\end{array}
$$

Charcoal, from Feature 46; interface between Bronze age cairn and Neolithic mound.

\section{BM-2009. Street House Farm}

$$
\mathbf{3 3 6 0} \pm \mathbf{5 0}
$$

Charcoal, from base $31 /$ interface 46 ; interface between Bronze age cairn and Neolithic mound.

\section{BM-2010. Street House Farm}

$$
\begin{array}{r}
\mathbf{3 1 7 0} \pm \mathbf{4 5} \\
\delta^{13} C=-24.0 \%
\end{array}
$$

Charcoal, from Feature 116, pit in center of mortuary enclosure. 
BM-2011. Street House Farm

$4630 \pm 80$

$\delta^{\circ} C=-24.2 \%$ clay mound.

BM-2012. Street House Farm

$4610 \pm 80$

Charcoal, ref 150, from Feature 105, old ground surface beneath clay mound.

BM-2013. Street House Farm

$4510 \pm 90$

Charcoal, from Feature 111, S sec of facade trench.

\section{BM-2014. Street House Farm}

$4630 \pm 70$

Charcoal, from Feature 110, S sec of facade trench.

BM-2060. Street House Farm

$4500 \pm 130$ enclosure.

$\delta^{13} \mathrm{C}=-25.6 \%$

\section{BM-2061. Street House Farm}

$\mathbf{5 0 7 0} \pm \mathbf{5 0}$

$\delta^{13} \mathrm{C}=-25.3 \%$

Charcoal, from Feature 99, proximal pit. Only timber found in situ in facade trench.

General Comment (BEV): BM-1966 to -1969 are from timbers of mortuary structure and form very consistent group within expected age range. BM-2013, -2014 are also consistent and confirm suggestion that facade timbers are roughly contemporary with those of mortuary structure. BM-2061, also from facade trench, was from largest timber on site (ca $\operatorname{lm}$ diam) and date may reflect initial age of wood. BM-2011, -2012 probably represent clearance or ritual activity prior to construction of mortuary structure. BM-2007 to -2009 represent interface between Neolithic structure and overlying Bronze age mound, although BM-2008 seems anomalous in this context. BM-2010 is from apparently sealed pit and does not accord with expectations. BM-2060 confirms charcoal underlying cairn represents activity on site ca time of construction of mortuary structure. Generally, results are extremely consistent and agree with present interpretation of site.

\section{Ascott-under-Wychwood series}

Collagen from bone samples from burials in separate stone cists in Neolithic long barrow at Ascott-under-Wychwood, Oxfordshire, England $\left(51^{\circ} 50^{\prime} \mathrm{N}, 1^{\circ} 35^{\prime} \mathrm{W}\right.$, Natl Grid Ref SP 299176). Coll 1968 and subm by D Benson, Oxford City and Co Mus (now Dyfed Archaeol Trust Ltd). 


\section{BM-1974. Ascott-under-Wychwood}

$4430 \pm 130$

Collagen from adult humerus, ref 534/37, from Deposit E, Chamber 7 .

$\delta^{13} \mathrm{C}=-21.4 \%$

BM-1975. Ascott-under-Wychwood

$\mathbf{3 4 8 0} \pm \mathbf{5 0}$

Collagen from adult femur, ref $330 / 5$, from thick deposit of bones piled outside and against outer stone of outer cist, sealing Abingdon bowl.

\section{BM-1976. Ascott-under-Wychwood}

$\mathbf{4 5 3 5} \pm \mathbf{4 0}$

Collagen from adult femur, ref $891 / 55$, from Deposit A, Chamber 3. General Comment (RB): cf BM-491b, $-492,-832,-833,-835$ to -837 and General Comment: R, 1976, v 18, p 19-20.

\section{BM-2018. Vale Castle}

$845 \pm 40$

Charcoal, ref D2B 7, from base of prehistoric rampart at Vale Castle, Guernsey, Channel Is $\left(49^{\circ} 35^{\prime} \mathrm{N}, 2^{\circ} 35^{\prime} \mathrm{W}\right.$, Natl Grid Ref WV 357817). Coll 1980 by K J Barton, Hampshire Mus and subm by I A Kinnes, Dept Prehist and Romano-British Antiquities, British Mus. Comment (RB): invalidated by misassoc.

\section{BM-2019. Jerbourg}

$\mathbf{1 3 0 0} \pm \mathbf{5 0 0}$

Charcoal, ref RIA(WIA), from lst rampart of defended site at Jerbourg, Guernsey, Channel Is $\left(49^{\circ} 30^{\prime} \mathrm{N}, 2^{\circ} 45^{\prime} \mathrm{W}\right.$, Natl Grid Ref $\mathrm{WV}$ 338751). Coll 1980 by R B Burns, Guernsey Mus, and subm by I A Kinnes. Fractionation correction estimated. Comment (RBB): sample, although small, was apparently stratigraphically secure in Early Bronze age context. No evidence of activity appropriate to this 1 st millennium $A D$ date was recovered, but result is clearly invalidated by misassoc.

\section{BM-2044. Canterbury}

$820 \pm 150$

Collagen from right femur of large domestic dog id by Juliet Clutton-Brock, Dept Zool, British Mus (Nat Hist), from articulated skeleton lying on thin layer of silt overlying tessellated floor of 2nd century AD Roman bldg and constituting primary fill of 7 th century Saxon hut, at Canterbury, Kent, England $\left(51^{\circ} 15^{\prime} \mathrm{N}, 1^{\circ} 5^{\prime} \mathrm{E}\right.$, Natl Grid Ref TR 150570). Coll 1978 by Marion Day and subm by T Tatton-Brown, Dir Canterbury Archaeol Trust. Comment (RB): cf BM-1523: $1850 \pm 70$, R, 1982, v 24, p 231-232. Disparity may be due to contamination of bone used for BM-1523, with ancient carbon during casting before destruction for dating. On hasis of new result, BM-2044 (bone not cast), skeleton may represent interment of remains of large Medieval hunting dog into Saxon levels during 12th century, and not redeposited Romano-British skeleton (see BM-1523, Comment, R, 1982, v 24, p 232); 12th century robber ditches on site (Tatton-Brown, pers commun). 


\section{Cyprus}

\section{Ayios Epiktitos Vrysi series}

Samples from ultimate and penultimate stages of late phase of Neolithic settlement (Peltenburg, 1975) at Ayios Epiktitos Vrysi, E of Kyrenia $\left(32^{\circ} 20^{\prime} \mathrm{N}, 33^{\circ} 25^{\prime} \mathrm{E}\right)$. Coll 1972 to 1973 and subm by E J Peltenburg, Univ Edinburgh.

\section{BM-1906. Ayios Epiktitos Vrysi}

Charcoal, ref VD 1, from pit and wall tumble.

$$
\begin{array}{r}
\mathbf{5 0 3 0} \pm \mathbf{8 0} \\
\delta^{13} C=-25.4 \% \text { o }
\end{array}
$$

BM-1907. Ayios Epiktitos Vrysi

$$
5120 \pm 45
$$

Charcoal, ref VE 8.

\section{BM-1908. Ayios Epiktitos Vrysi}

Charcoal, ref VD 10.

General Comment (EJP): BM-1906, -1907 provide dates for accumulation of debris forming late phase at Vrysi; BM-1908 dates immediately preceding levels. BM-1906 is particularly important in determining pace of developments during transition from Neolithic to Chalcolithic Cyprus. For other dates from site, $c$ B Birm-182: $3875 \pm 145$, R, 1971, v 13, p 155; Birm-337: $3790 \pm 140, \mathrm{R}, 1973$, v 15, p 468; GU-453: $3468 \pm 79$, -454: $3401 \pm 94,-455: 3303 \pm 150$, R, 1973, v 15, p 489; BM-843: $3405 \pm 67$, $-844: 3325 \pm 47,-845: 3410 \pm 57, \mathrm{R}, 1977, \mathrm{v} 19, \mathrm{p} 146$.

\section{Ecuador}

\section{Hacienda Guarumel series}

Samples from layers corresponding to occupation horizons, in shell midden of Jambeli culture, at coastal site Hacienda Guarumel, Canal de Jambeli, near Machala, Azuay $\left(3^{\circ} 20^{\prime} \mathrm{S}, 80^{\circ} 0^{\prime} \mathrm{W}\right)$. No ${ }^{14} \mathrm{C}$ dates available for culture, which represents small-scale exploitation of coastal resources; culture is provisionally dated to $500 \mathrm{BC}$ to AD 500. Coll 1976 and subm by Elizabeth J Carter, Inst Archaeol, Univ London.

\section{BM-1688. Hacienda Guarumel} Charcoal, ref HG 76 A4 S22.

\section{BM-1689. Hacienda Guarumel}

Charcoal, ref HG 76 B27 S52.

\section{Egypt}

\section{BM-1846. Gawāsīs}

Knot of grass rope (Desmostachya sp id by D Cutler, Royal Botanic Gardens, Kew), from shrine of Ankhow at mouth of Wādī Gawāsīs, N of Quseir, on Red Sea coast $\left(26^{\circ} 40^{\prime} \mathrm{N}, 34^{\circ} 10^{\prime} \mathrm{E}\right)$ assoc with Pharaonic port 
and texts of Sesostris I (Sayed, 1977; 1978; 1980). Coll 1976 and subm by A Sayed, King Abdulaziz Univ, Jeddah. Comment (RB): measured as check on BM-1844: $3230 \pm 45, \mathrm{R}, 1982$, v 24, p 276.

\section{Montgaudier series}

\section{France}

Samples from Magdalenian levels in different sites within or adjacent to very large multi-chambered caves (Duport, 1976) at Montgaudier Cave, near Montbron, Charente (45 $\left.40^{\prime} \mathrm{N}, 0^{\circ} 30^{\prime} \mathrm{E}\right)$. Coll 1975 to 1980 by L Duport, Angoulême and subm by G de G Sieveking, Dept Prehist and Romano-British Antiquities, British Mus.

\section{BM-1911. Montgaudier}

$11,450 \pm 70$

Collagen from bone fragments, from Abri Gaudry, Sq A'44, Layer 2.
BM-1912. Montgaudier
$12,180 \pm 130$

Collagen from bone fragments, from Abri Gaudry, Sq D'43, Layer 1 .
BM-1913. Montgaudier
$18,050 \pm 230$
$18,050 \pm 230$
$\delta^{13} C=-20.4 \%$ o

Collagen from bone fragments, from exterior platform.
BM-1914. Montgaudier
$18,180 \pm 1070$
Collagen from bone fragments, from opposite hearth, Sq J 20.

\section{BM-1916. Montgaudier}

$\mathbf{1 3}, \mathbf{3 2 0} \pm \mathbf{3 6 0}$

Collagen from bone fragments, from Abri Paignon.

General Comment (GdeGS): BM-1911, -1912 are from superimposed final Magdalenian occupation deposits in Abri Gaudry. Results are in stratigraphic order and are closely similar to other determinations for final Magdalenian in France. BM-1913 dates bone fragments assoc with Magdalenian artifacts, on platform in front of main entrance to cave, where 19th century excavations uncovered decorated baton and other Magdalenian IV material. This result is much earlier than any other Magdalenian IV date and seems to suggest that early Magdalenian deposits underlie those of Magdalenian IV here. BM-1914, from bone assoc with Magdalenian deposits from lst floor level in cave, suggests that deposits are also of early Magdalenian date.

\section{Figure of Christ series}

Wood samples (Juglans sp, id by Rowena Gale, Royal Botanic Gardens, Kew) drilled from detachable arms and body of near lifesize carved wooden figure of Christ, of French origin and claimed to date to 12 th century AD. Subm 1981 by $N$ M Stratford, Dept Medieval and Later Antiquities, British Mus. 
BM-1977. Figure of Christ

Wood drilled from center of body at back of figure.

BM-1978. Figure of Christ

Wood drilled from left arm.

BM-1979. Figure of Christ

Wood drilled from right arm.

\section{Agios Petros series}

\section{Greece}

Samples from Level 7 in Neolithic settlement site at Agios Petros, Kyra Panagia, N Sporades $\left(39^{\circ} 20^{\prime} \mathrm{N}, 24^{\circ} 0^{\prime} \mathrm{E}\right)$. Coll 1981 and subm by $\mathrm{N}$ Efstratiou, Inst Archaeol, Univ London. Site is earliest settlement found in Aegean region (Evans and Renfrew, 1968).

\section{BM-2020. Agios Petros}

Charcoal from $\mathrm{W}$ of Wall $\mathrm{K}$.

\section{BM-2021. Agios Petros}

$6400 \pm 80$ $\delta^{13} C=-24.8 \%$ o

$$
\mathbf{5 5 1 0} \pm \mathbf{3 9 0}
$$

$\delta^{13} \mathrm{C}=-25.0 \%$

General Comment (NE): dates are as expected; BM-2020 is well within Middle Neolithic period of Greek sequence while BM-2021 comes from disturbed deposit and is understandly quite late.

\section{E Hungarian Neolithic series}

\section{Hungary}

Samples dated to establish Neolithic sequence as part of Hungarian Acad Sci Topographic Program in Ko Békés II, E Hungary. Coll by Dr J Makkay, Hungarian Acad Sci and subm by Dr J Chapman, Univ Newcastle upon Tyne.

BM-1860. Békésszentandrás no. 28

$$
\begin{array}{r}
\mathbf{6 0 8 0} \pm \mathbf{6 0} \\
\delta^{13} C=-22.6 \%
\end{array}
$$

Charcoal from 80 to $160 \mathrm{~cm}$ level of late Alföld Bandkeramik refuse pit at Békésszentandrás no. 28, $5 \mathrm{~km}$ SSE of Békésszentandrás, Ko Békés $\left(46^{\circ} 50^{\prime} \mathrm{N}, 20^{\circ} 30^{\prime} \mathrm{E}\right)$. Coll 1978.

\section{BM-1861. Szarvas no. 1}

$$
5630 \pm 140
$$

Charcoal from depth 160 to $190 \mathrm{~cm}$ in Szakalhát period level at Szarvas no. 1, $1 \mathrm{~km} \mathrm{NW}$ of Szarvas, Ko Békés $\left(46^{\circ} 55^{\prime} \mathrm{N}, 20^{\circ} 30^{\prime} \mathrm{E}\right)$. Coll 1975.

\section{BM-1862. Battonya-Basarága}

$6580 \pm 60$

Charcoal from fill of Körös culture refuse Pit no. III at BattonyaBasarága, near Battonya, Ko Békés $\left(46^{\circ} 15^{\prime} \mathrm{N}, 21^{\circ} 0^{\prime} \mathrm{E}\right)$. Coll 1977. 


\section{Endröd no. 39 series}

Samples from fill of Körös culture refuse pits at Endröd no. 39, I of largest early Neolithic sites in Békés II area, $4.5 \mathrm{~km}$ SSW of Ko Békés $\left(46^{\circ} 55^{\prime} \mathrm{N}, 20^{\circ} 45^{\prime} \mathrm{E}\right)$. Coll 1975 to 1976.

BM-1863. Endröd no. 39

$6840 \pm 110$

Charcoal from Pit no. IV/I.

$\delta^{13} C=-26.3 \%$

BM-1868. Endröd no. 39

$6830 \pm 60$

Charcoal from Pit no. I, Tr XVIII, at depth 60 to $90 \mathrm{~cm}$.

BM-1870. Endröd no. 39

$6600 \pm 80$

Charcoal from Pit no. I, Tr XVIII, at depth 90 to $120 \mathrm{~cm}$.

BM-1871. Endröd no. 39

Charcoal from Pit no. I, Tr XIX.

BM-1864. Endröd no. 35

Charcoal from fill of Körös refuse Pit no. III, at Endröd no. $35,3 \mathrm{~km}$

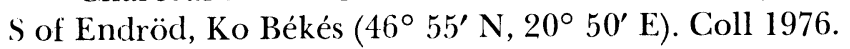

Szarvas no. 23 series

Samples from Körös culture refuse pits at Szarvas no. $23,1.5 \mathrm{~km} \mathrm{E}$ of Szarvas, Ko Békés $\left(46^{\circ} 50^{\prime} \mathrm{N}, 20^{\circ} 35^{\prime} \mathrm{E}\right.$ ), assoc with pottery possibly dating to earliest Neolithic occupation of Hungary. Coll 1976.

BM-1865. Szarvas no. 23

$6190 \pm 140$

Charcoal from fill of Pit no. VII/I.

BM-1866. Szarvas no. 23

Charcoal from fill of Pit no. IX/I.

\section{BM-1867. Szarvas no. 102}

Charcoal from inside House 1 of classic Alföld Bandkeramik period at Szarvas no. 102, 2km NNE of Szarvas, Ko Békés $\left(46^{\circ} 55^{\prime} \mathrm{N}, 20^{\circ} 30^{\prime} \mathrm{E}\right)$. Coll 1977.

General Comment (JC): BM-1863, -1868, -1870, -1871 agree well with middle to late Körös stylistic dating. BM-1863 and -1868 provide 1st dates for middle Körös phase, whilst BM-1870 and -1871 agree well with late Körös dates from Deszk, Hódmeźóvásárhely-Kotacpart (Bln-75: 7090 $\pm 100 \mathrm{bp}, \mathrm{R}, 1964$, v 6, p 315), Devavanya-Atyaszeg (6190 bp; Kalicz and Makkay, 1977, p 110) and BM-1862 from Battonya. BM-1865 and -1866 agree with late-latest Körös dates from Deszk (Bln-581 to -584: 6605-62.60 bp, R, 1970, v 12, p 408); Szarvas-23 Pits VIII and IX contain 
late Körös material in assoc with early AVK pottery. BM-1864 is 300 to $400 \mathrm{yr}$ too young. BM-1867 seems 300 to $400 \mathrm{yr}$ too young for a classic Alföld Bandkeramik phase ( $c f$ date for Tarnabod, Bln-123: $6280 \pm 100$ bp, R, 1964, v 6, p 316). BM-1860 fits well with late Alföld Bandkeramik dates of $6136 \pm 100$ bp from Szamossályi (Bln-404, R, 1966, v 8, p 39), $6180 \pm 100 \mathrm{bp}$ from Ostoros (Bln-549, R, 1970, v 12, p 410) less well with Bln-505 from Tiszavasvari-Keresztfal $(6305 \pm 100 \mathrm{bp}, \mathrm{R}, 1970, \mathrm{v} 12$, p 413). BM-1861 appears to be 400 to $500 \mathrm{yr}$ too young and may date 4 th millennium Tisza occupation on site.

\section{Pakistan series}

\section{Pakistan}

Charcoal samples measured to provide information on previously undated periods in Pakistan (Durrani, 1981; 1982; Khan, 1981; Allchin, 1982). All samples coll 1980 to 1981 and subm by K D Thomas, British Archaeol Mission to Pakistan, and Inst Archaeol, Univ London.

\section{Sarai Khola series}

Samples from site at Sarai Khola, Taxila Valley, Taxila, Punjab Prov $\left(33^{\circ} 30^{\prime} \mathrm{N}, 72^{\circ} 30^{\prime} \mathrm{E}\right)$. BM-1934, -1935, -1937, -1939, -1940 all from Neolithic contexts from floors assoc with degraded mud-brick and ash pits. These levels are only manifestations of this culture in the Punjab. No previous dates for Neolithic in Pakistan $E$ of $R$ Indus. BM-1936, -1938, -1942 to -1946 from Kot Dijian levels. BM-1947 from Medieval level.

\section{BM-1934. Sarai Khola}

Charcoal, ref SK 81/4.

BM-1935. Sarai Khola

Charcoal, ref SK 81/3.

BM-1936. Sarai Khola

Charcoal, ref SK C8.

BM-1938. Sarai Khola

Charcoal, ref SK C6.

BM-1939. Sarai Khola

Charcoal, ref SK 81/6.

BM-1940. Sarai Khola

Charcoal, ref SK 81/5.

BM-1942. Sarai Khola

Charcoal, ref C4.

$$
\begin{array}{r}
4250 \pm 110 \\
\delta^{13} C=-23.7 \% \text { o }
\end{array}
$$

$$
4140 \pm 230
$$$$
\delta^{13} \mathrm{C}=-23.5 \% \text { o }
$$

$3890 \pm 230$

$\delta^{13} C=-24.6 \%$ o

$$
3810 \pm 60
$$$$
\delta^{13} C=-24.6 \% \text { o }
$$

$$
4310 \pm 120
$$$$
\delta^{13} C=-25.2 \% \text { o }
$$

$$
4380 \pm 170
$$$$
\delta^{13} C=-24.2 \% \text { o }
$$

$$
3910 \pm 70
$$

$\delta^{13} C=-24.5 \%$ 。 
BM-1943. Sarai Khola

Charcoal, ref SK81/2.

BM-1944. Sarai Khola

Charcoal, ref C5.

BM.1945. Sarai Khola

Charcoal, ref SK81/1.

BM-1946. Sarai Khola

Charcoal, ref C1.

BM-1947. Sarai Khola

Charcoal, ref C7.

BM.1941. Islam Chauki
$3700 \pm 60$

$\delta^{13} C=-24.9 \%$

$4040 \pm 200$

$\delta^{13} C=-26.1 \%$

$3790 \pm 60$

$\delta^{13} C=-24.7 \%$

$\mathbf{3 7 0 0} \pm \mathbf{8 0}$

$\delta^{13} C=-23.1 \%$

$$
\mathbf{8 7 0} \pm \mathbf{5 0}
$$$$
\delta^{13} C=-24.5 \%
$$

$3690 \pm 450$

$\delta^{13} C=-24.7 \%$

Kot Dijian level sealed by floors and degraded mud-brick at Islam Chauki, W of Bannu City, North West Frontier Prov $\left(33^{\circ} 0^{\prime} \mathrm{N}, 70^{\circ} 30^{\prime} \mathrm{E}\right)$.

\section{Hathial West series}

Samples from well-sealed floor levels of Kot Dijian period at Hathial West, near Taxila Mus, Punjab Prov ( $\left.33^{\circ} 30^{\prime} \mathrm{N}, 72^{\circ} 30^{\prime} \mathrm{E}\right)$.

\section{BM-1948. Hathial West}

Charcoal, ref 1.

\section{BM-1949. Hathial West}

Charcoal, ref 2.

\section{BM-1950. Hathial North}

$$
\begin{array}{r}
\mathbf{3 6 0 0} \pm \mathbf{6 0} \\
\delta^{13} C=-25.4 \% \\
\mathbf{3 7 5 0} \pm \mathbf{1 0 0} \\
\delta^{13} C=-25.2 \% 0
\end{array}
$$

$$
1740 \pm 40
$$

$\delta^{13} C=-24.4 \%$ o

Charcoal from under structural materials assoc with fortification of early Historic period at Hathial North, near Taxila Mus, Punjab Prov $\left(33^{\circ} 30^{\prime} \mathrm{N}, 72^{\circ} 30^{\prime} \mathrm{E}\right)$, assoc with early Historic period and unique grayware pottery.

\section{Bhir Mound series}

Samples from major occupation levels from one of Historic period cities of Taxila Valley at Bhir mound, Taxila, Punjab Prov $\left(33^{\circ} 30^{\prime} \mathrm{N}\right.$, $\left.72^{\circ} 30^{\prime} \mathrm{E}\right)$.

BM-1951. Bhir Mound

$1990 \pm 60$

Charcoal, ref 1.

$\delta^{13} C=-26.5 \%$ o 


\section{BM-1952. Bhir Mound}

Charcoal, ref 2.

BM-1953. Bhir Mound

Combined charcoal samples, refs 3 and 4.

BM-1954. Bhir Mound

Charcoal, ref 5.

BM-1955. Bhir Mound

Charcoal, ref 6.

BM-1956. Bhir Mound

Charcoal, ref 7 .

BM-1957. Bhir Mound

Charcoal, ref 8.

BM-1958. Bhir Mound

Charcoal, ref 9.

BM-1959. Bhir Mound

Charcoal, ref 10.

BM-1960. Bhir Mound

Charcoal, ref 11.

BM-1961. Bhir Mound

Charcoal, ref 12.

BM-1963. Bhir Mound

Charcoal, ref 14.

BM-1964. Bhir Mound

Charcoal, ref 15.

BM-1965. Bhir Mound

Charcoal, ref 16.
$1920 \pm 170$

$\delta^{13} C=-24.7 \%$ o

$1930 \pm 50$

$\delta^{13} \mathrm{C}=-25.2 \%$

$1830 \pm 40$

$\delta^{13} C=-23.5 \%$ o

$2050 \pm 60$
$\delta^{13} C=-25.2 \%$

$1795 \pm 35$

$\delta^{13} \mathrm{C}=-24.8 \%$

$2000 \pm 45$

$\delta^{13} C=-24.1 \%$ 。

$2010 \pm 40$

$\delta^{13} C=-24.4 \%$

$1950 \pm 50$

$\delta^{13} C=-24.7 \%$

$1805 \pm 35$

$\delta^{13} C=-24.7 \%$

$2050 \pm 80$

$\delta^{1.3} C=-25.2 \%$

$2120 \pm 200$

$\delta^{13} C=-25.4 \%$ 。

$2080 \pm 80$

$\delta^{13} C=-24.2 \%$

$2090 \pm 90$

$\delta^{13} C=-25.1 \%$

\section{Rehman Dheri series}

Samples from Kot Dijian III layers at Rehman Dheri, $23 \mathrm{~km} \mathrm{~N}$ of Dera Ismail Khan City, North West Frontier Prov $\left(31^{\circ} 50^{\prime} \mathrm{N}, 70^{\circ} 55^{\prime} \mathrm{E}\right)$. 
BM-2062. Rehman Dheri

Charcoal, ref RHDl, from depth $170 \mathrm{~cm}$.

\section{BM-2063. Rehman Dheri}

Charcoal, ref RDH2, from depth $50 \mathrm{~cm}$.

General Comment (KDT): BM-1934, -1935, -1937, -1940, all from Neolithic contexts, are comparable with dates from sites W of R Indus, Ghaligai, Swat and Burzahom, Kashmir (Agrawal, 1982, p 270-271), but are later than those from Kili Ghul Mohammad (Agrawal, 1982, p 270) and Mehrgarh (Lechevallier and Quivron, 1981, p 91) both in Baluchistan. Results are close to dates from early Kot Dijian levels (see below) and suggest that Sarai Khola was not abandoned for long period between Neolithic and Kot Dijian occupations. BM-1936, -1942, -1944, relating to early Kot Dijian pits at Sarai Khola, and BM-1938, -1945, from upper fills of these pits, are rather later than expected, clustering in late Kot Dijian when compared with dates from Kot Diji (Khan, 1981), Gumla (Dani, 1971), Amri (Casal, 1964) and Rehman Dheri III (Durrani, 1981; 1982, but see BM-2062, -2063, below). Other dates from Kot Dijian contexts, BM-1943, -1946 from later floors at Sarai Khola, BM-1941 from Islam Chauki, BM-1948, -1949 from Hathial West and BM-2062, -2063 from Rehman Dheri III form remarkably consistent homogeneous group that compares well with dates from Tarakai Kala Dheri (BM-1690 to $-1695, \mathrm{R}, 1982$, v 24, p 281). All these dates indicate survival of Kot Dijian elements into latter part of 3rd millennium BC and are much later than other Kot Dijian sites in Pakistan, falling into period of mature Harappan. A reappraisal of chronol, cultural, and geographic relationship of Kot Dijian and Harappan "cultures" is indicated; it may be significant that, with the exception of small mound of Hisam Dheri near Rehman Dheri (Dani, 1971), no Harappan sites are known in vicinity of Kot Dijian sites discussed here. Dates for Hathial North (BM-1950) and Bhir Mound (BM-1951 to -1965) all relate to Historic period. BM-1950 appears considerably later than date suggested by assoc pottery (Allchin, 1982, p 13). Bhir Mound, till recently believed to be lst city in Taxila but now replaced by Hathial (Allchin, 1982), remains one of most important archaeol sites in Pakistan. Samples were coll from small excavation adjacent to extensive excavations of Sharif in 1969. BM-1961 to -1965, from series of clearly stratified floors of earliest occupation, have produced samples much later than expected and may indicate that this part of city was built quite late and persisted during occupation at Sirkap, previously held to succeed Bhir Mound (Marshall, 1951). BM-1951 to -1960 are apparently assoc with industrial activity, indicated by large amounts of charcoal and iron slag. Range of dates may reflect age of wood exploited but most dates are late, clustering in lst century AD after calibration. Date as late as $1740 \pm 40$ (BM-1950) is quite unexpected and it seems clear that chronol of site and others in Taxila will have to be reconsidered. BM-1947, from rubbish 
pit in Phase IV at Sarai Khola is acceptable for late Hindu Shahi site E of R Indus (Halim, 1972a,b, p 112).

Peru

BM-1633. Cusichaca project

$2380 \pm 70$

Charcoal, ref Q211-75-Level 12, from Bldg 17, Group 3 of prom ontory site overlooking $\mathrm{R}$ Cusichaca, near confluence with $\mathrm{R}$ Urubamba, Chuncuchua, Huillca Raccay, Dept Cuzco, Peru $\left(13^{\circ} 10^{\prime} \mathrm{S}, 72^{\circ}\right.$ 25' W). Coll 1975 and subm by Ann Kendall, Dir Cusichaca Archaeol Proj. Sample from $1 \mathrm{~m}$ depth in test pit, occupation level assoc with Chanapata pottery (Kendall, 1976). Comment (AK): result fits well within broad age range of 1000 to $300 \mathrm{BC}$ expected.

\section{Moncín series}

\section{Spain}

Samples from clearly stratified Bronze-age midden $2.2 \mathrm{~m}$ deep, overlying stone wall, at multi-period settlement site at Moncín (MorenoLopez, Legge, and Harrison, in press) Borja, Zaragoza $\left(41^{\circ} 50^{\prime} \mathrm{N}, 1^{\circ}\right.$ $30^{\prime} \mathrm{W}$ ). Houses on stone terraces and Bell Beaker and Early Bronze-age cultural material present on site. Coll 1980 by R J Harrison, Dept Classics and Archaeol, Univ Bristol and subm by R J Harrison and A J Legge, Extra Mural Dept, Univ London.

BM-1921. Moncín

$2960 \pm 40$

$\delta^{13} \mathrm{C}=-23.2 \%$

Charcoal, ref 1, (Period III), from fill of large pit, F2, cutting into earlier midden deposit. Assoc with animal bone and pottery.

BM-1925. Moncín

Charcoal, ref 2, (Period III), from fill of large pit, F3, similar to F2.

\section{BM-1926. Moncín}

Charcoal, ref 3, (Period II), from Layer 3.

\section{BM-1927. Moncín}

Charcoal, ref 5, (Period I), from Layer 6.

\section{BM-1928. Moncín}

Charcoal, ref 4, (Period II), from Level 7.

$$
\begin{array}{r}
\mathbf{3 0 2 0} \pm \mathbf{4 5} \\
\delta^{13} \mathrm{C}=-25.0 \%
\end{array}
$$

$\mathbf{2 8 8 0} \pm \mathbf{3 5}$ $\delta^{13} C=-25.1 \%$

$$
\begin{array}{r}
\mathbf{3 0 4 0} \pm \mathbf{4 5} \\
\delta^{13} C=-28.7 \%
\end{array}
$$

$$
2915 \pm 45
$$$$
\delta^{13} C=-23.8 \%
$$

General Comment $(\mathrm{RJH})$ : examination of archaeol material from midden shows it to be important and homogeneous deposit of beginning of Spanish Late Bronze age, and characteristic Boquique decorated sherds agree exactly with ${ }^{14} \mathrm{C}$ dates. Midden is last phase of 1200 -yr occupation of site, which stretches from 2200 to 1000 BC. Culturally, material is analogous to Valencian Bronze age of Spanish Levant. 


\section{Ferrandell-Oleza series}

Samples from Beaker settlement site at Ferrandell-Oleza, Old Settlement, Valldemosa, Mallorca, Baleares $\left(39^{\circ} 40^{\prime} \mathrm{N}, 2^{\circ} 30^{\prime} \mathrm{E}\right)$, assoc with Beaker pottery, worked flints and domestic animal remains. Coll 1981 and subm by $\mathrm{W} \mathrm{H}$ Waldren, Donald Baden-Powell Quaternary Res Centre, Pitt Rivers Mus, Univ Oxford and Dir, Deya Archaeol Mus and Research Centre, Deya de Mallorca.

\section{BM-1981. Ferrandell-Oleza}

$3720 \pm 35$

Charcoal, from Exploratory Tr S, Sec Q-J3, assoc with flint blades and Beaker sherds.

\section{BM-1982. Ferrandell-Oleza}

$$
1710 \pm 60
$$

Charcoal, from Structure C2, from under renovated pebble floor. General Comment (RB): cf BM-1843: $3950 \pm 60$, R, 1982, v 24, p 282, (see also Waldren, 1981a; b). BM-1982 is ca $2000 \mathrm{yr}$ later than expected and appears to be invalidated by misassoc.

\section{Muertos Gallard series}

Samples from Beaker occupation of rock shelter at Muertos Gallard, Deya, Mallorca, Baleares $\left(39^{\circ} 40^{\prime} \mathrm{N}, 2^{\circ} 35^{\prime} \mathrm{E}\right)$. Coll 1967 and subm by W $\mathrm{H}$ Waldren.

BM-1993. Muertos Gallard

Charcoal, ref 2 and 3, from under ' $F$ ' rock, $90 \mathrm{~cm}$ level.

\section{BM-1994. Muertos Gallard}

Charcoal, ref 19, from Sec J6, $160 \mathrm{~cm}$ level.

$$
\delta^{13} C=-24.2 \%
$$

General Comment (RB): cf Y-1789: $3790 \pm 80$ (Waldren, 1981a); BM1994 is much earlier than expected, BM-1993 is invalidated by misassoc.

\section{BM-1995. Son Matge}

$$
\begin{array}{r}
\mathbf{3 3 8 0} \pm \mathbf{5 0} \\
\delta^{13} C=-24.0 \% 0
\end{array}
$$

Charcoal from $\operatorname{Tr} 3 \mathrm{C}, 240 \mathrm{~cm}$ level, at rock shelter at Son Matge, Estret, Valldemosa, Mallorca, Baleares (39 $\left.35^{\circ} \mathrm{N}, 2^{\circ} 25^{\prime} \mathrm{E}\right)$. Coll 1975 and subm by W $\mathrm{H}$ Waldren. Comment (RB): date is late Pretalayotic/ early Talayotic (for other dates for Son Matge, see Waldren, 1981a).

BM-1998. Son Puig-Servera

$2645 \pm 40$ $\delta^{13} C=-24.2 \%$

Charcoal from habitation level at Son Puig-Servera, Palma, Mallorca, Baleares $\left(39^{\circ} 35^{\prime} \mathrm{N}, 2^{\circ} 30^{\prime} \mathrm{E}\right)$. Coll 1967 by $\mathrm{G}$ Rossello-Bordoy, Dir Mus Mallorca, Palma and subm by W H Waldren. Comment (RB): cf Y-2673: $2180 \pm 100$ (Waldren, 1981a). 


\section{Taula de Torralba d'en Salord series}

Samples from 3 distinct levels in interior ( $\mathrm{E}$ part of bldg) of Taula de Torralba d'en Salord, $2.5 \mathrm{~km}$ SE of Alayor, Menorca, Baleares $\left(39^{\circ}\right.$ $55^{\prime} \mathrm{N}, 4^{\circ} 10^{\prime} \mathrm{E}$ ). Coll 1981 and subm by E A C Sanders, Deya Archaeol Mus and Research Centre. Samples measured to verify dating based on ceramics and coins (BM-2003), to establish time of abandonment of Taula (BM-2004; also provides min date for introduction to Menorca of shrew, Crocidura sp, not previously found in archaeol context there), and to help resolve disputed question of whether Taula was roofed (BM-2005).

Charcoal from Sec Entrada A, Level Lower IV $(125 \mathrm{~cm})$, representing period of continual (probably ritual) use of Taula. Ceramics and 3 of 4 coins from this level indicate date of 230 to $150 \mathrm{BC}$; 4 th coin was minted in Roman colony of Nîmes ca 0 BC and may be intrusive.

\section{BM-2004. Taula de Torralba d'en Salord}

$1890 \pm 35$

Charcoal from Sec M, Level Upper IV (90 to $95 \mathrm{~cm})$. End of period of (ritual) use of Taula (abandonment of bldg); 1st level containing remains of shrew, Crocidura sp.

\section{BM-2005. Taula de Torralba d'en Salord $\delta^{13} C=-24.5 \%$}

Combined charcoal samples from Sec M, Level III $(80 \mathrm{~cm})$ and Sec K, Level III $(85 \mathrm{~cm})$. Level III covers lower levels preserving many whole artifacts, indicating rapid sedimentation following abandonment of Taula (possibly following collapse of roof). If bldg was roofed as has been suggested and sample represents collapsed and burned roof timbers, date should be earlier than underlying level Upper IV (BM-2004).

General Comment (RB): BM-2003 tends to support earlier dating for period of use of Taula of 230 to $150 \mathrm{BC}$ based on ceramics and coins, but at $+1 \sigma$ does not prove later Roman coin is intrusive (cf also, BM-2004). BM-2004 provides date for abandonment of Taula and this appears later than dates of 2 major hist events suggested as possible causes of abandonment (last Punic war, $146 \mathrm{BC}$ and Roman occupation of Balearic Is, $123 \mathrm{BC})$. Result also dates lst recorded occurrence in archaeol levels on Menorca of remains of shrew Crocidura sp, now numerous on $\mathrm{I}$. BM-2005 does not support suggestion that Taula was roofed (while leaving open possibility that bldg was roofed and remains of roof are not represented by this sample).

\section{Tell Brak series}

$$
\text { Syria }
$$

Samples from occupation site at Tell Brak (Mallowan, 1947; Oates, 1977; 1982), near El Haseke, NE Syria $\left(36^{\circ} 40^{\prime} \mathrm{N}, 41^{\circ} 0^{\prime} \mathrm{E}\right)$. Coll 1981 and subm by Joan Oates, Girton Coll, Univ Cambridge. All samples 
except BM-1970 from destruction level preceding construction of Agade "palace" by Naram-Sin ca 2400 to 2225 BC.

BM-1970. Tell Brak

$\mathbf{3 4 4 0} \pm \mathbf{5 0}$

$\delta^{13} C=-24.2 \%$ o

Wood charcoal from Tr F5, Loc 29 (late Agade/early Ur III).

BM-1971. Tell Brak

$3590 \pm 50$

$\delta^{13} C=-22.7 \%$ 。

Burned grain sample scraped from jar in room in $\operatorname{Tr} \mathrm{CH}$, Loc 450.

BM-1972. Tell Brak

$3620 \pm 50$

$\delta^{13} \mathrm{C}=-24.2 \%$ 。

Burned grain sample scraped from different jar in same room as BM-1971 above.

\section{BM-1973. Tell Brak}

$3590 \pm 45$

Wood charcoal from Tr CH, Loc 445.

$\delta^{13} C=-24.1 \%$ o

General Comment (RB): dates when calibrated are ca 250 yr later than expected for Agade destruction (са 2300 вс) and late Agade/early Ur III (ca $2100 \mathrm{BC}$ ), but agree with previous measurements ( $\mathrm{R}, 1982, \mathrm{v} 24$, p 285).

\section{Thailand}

\section{BM-2016. Ban Don Ta Phet}

$1810 \pm 210$

$\delta^{13} C=-25.0 \%$ o

Sample of carbon extracted by acid treatment from pottery sherds 413555 and 157865 7, from Ban Don Ta Phet, Phanom Thuan dist, Kanchanaburi prov, SW Thailand (14 $\left.{ }^{\circ} 15^{\prime} \mathrm{S}, 99^{\circ} 45^{\prime} \mathrm{E}\right)$. Coll 1981 by I Glover, Inst Archaeol, Univ London and subm by M Cowell, Research Lab, British Mus following failure of thermoluminescence dating due to radon loss. Comment (RB): result agrees broadly with archaeol date expected (Rajpitak and Seeley, 1979), but possible sources of error are contamination by ancient organic carbon from clay body of sherd (probably less than $10 \%)$ and fractionation $\left(\delta^{13} \mathrm{C}\right.$ estimated).

\section{REFERENCES}

Agrawal, D P, 1982, The archaeology of India: Scandinavian Inst Asian Studies mon no. 46, London and Malmö, Curzon Press.

Allchin, F R, 1982, How old is the city of Taxila?: Antiquity, v 56, p 8-14.

Barrett, J, Bradley, R, Green, M, and Lewis, B, in press, The earlier prehistoric settlement of Cranborne Chase: Antiquaries Jour, v 62, p 00-00.

Brisay, K, de, 1972, Preliminary report on the exploration of the Red Hill at Osea Road, Maldon, Essex: Colchester Archaeol Group Bull, v 15, p 24-43. 1973, A further report on the excavations of the Red Hill at Osea Road, Maldon: Colchester Archaeol Group Bull, v 16, p 20-36.

Casal, J M, 1964, Fouilles d'Amri: Paris, Lib C Klincksieck.

Clark, R M., 1975, A calibration curve for radiocarbon dates: Antiquity, v 49, p 251-266. Clutton-Brock, J and Burleigh, Richard, in press, Some archaeological applications of the dating of animal bone by radiocarbon with particular reference to postPleistocene extinctions, in Internatl symposium on C-14 and Archaeol, 1st, Gronirigen, Netherlands, 24-28 Aug, 1981, Proc: PACT Jour, in press.

Dani, A H 1971, Excavations in the Gomal Valley: Ancient Pakistan, v 5, p 1-177.

Duport, L, 1976, La grotte de Montgaudier, in Union Internatl Sci Préhist et Protohist (UISPP) Cong, 9th, Nice, 13-18 Sept, 1976: Livret-Guide de l'excursion A4 SudOuest (Aquitaine et Charente), p 151-158. 
Durrani, F A, 1981, Indus civilization, evidence west of Indus, in Dani, A H, ed, Indus civilization, new perspectives: Islamabad, Centre Study Civilization Central Asia, p 133-138.

1982, Rehman Dheri and the birth of civilization in Pakistan: Univ London Inst Archaeol Bull, no. 18, p 191-207.

Evans, J D and Renfrew, C, 1968, Excavations at Saliagos near Antiparos: Athens, British School Archaeol.

Green, C J S, 1974, Interim report on excavations at Poundbury, Dorchester 1973: Dorset Nat Hist and Archaeol Soc Proc, v 95, p 97-100.

Grigson, C, 1978, The late glacial and early Flandrian ungulates of England and Wales-an interim review, in Limbrey, S and Evans, J G, eds, The effect of man on the the landscape-the lowland zone: CBA research rept 21, p 46-56. in press, The horn core of an aurochs (Bos primigenius) from Corhampton, Hampshire: Hampshire Field Club Proc, in press.

Halim, M A, 1972a, Excavations at Sarai Khola. Pt I: Pakistan Archaeol, v 1, p 23-89. 1972b, Excavations at Sarai Khola. Pt II: Pakistan Archaeol, v 8, p 1-112.

Harman, M, Molleson, T I, and Price, J L, 1981, Burials, bodies and beheadings in Romano-British and Anglo-Saxon cemeteries: British Mus (Nat Hist) Bull (geol ser), v 35, pt 3, p 145-188.

Jones, R L, ms, 1971, A contribution to the late Quaternary ecological history of Cleveland, NE Yorkshire: Unpub PhD thesis, Univ Durham.

Kalicz, N and Makkay, J, 1977, Die Linienbankeramik in der Grossen Ungarischen Tiefebene: Budapest, Akad Kiado.

Kendall, A, 1976, Ceramic data of the Urubamba Valley, Cuzco: Baessler-Arkhiv (ns), v $24, \mathrm{p}$ 47-52.

Khan, F A, 1981, Kot Diji culture, its greatness, in Dani, A H, ed, Indus civilization, new perspectives: Islamabad, Centre Study Civilization Central Asia, p 15-24.

Lechevallier, M and Quivron, G, 1981, The Neolithic in Baluchistan - new evidence from Mehrgarh, in Hartel, H, ed, South Asian archaeology 1979: Berlin, Dietrich Reimer Verlag, p 71-92.

Mallowan, M E L, 1947, Excavations at Tell Brak and Chagar Bazar: Iraq, v 9, p 1-258.

Marshall, Sir J, 1951, Taxila: Cambridge, Cambridge Univ Press.

Moreno-Lopez, G, Legge, A J, and Harrison, R J, in press, Avance sobre las excavaciónes arqueológicas en Moncín, Borja (Prov Zaragoza): Cuadernos Estudios Borjanos, $\mathrm{v} 7$, in press.

Oates, D, 1977, The excavations at Tell Brak, 1976: Iraq, v 39, p 233-244.

1982, Tell Brak, in Curtis, J, ed, Fifty years of Mesopotamian discoverythe work of the British School of Archaeology in Iraq 1932-1982: London, British School Archaeol in Iraq (Gertrude Bell Memorial), p 62-71.

Payne, S, 1981, Notes on the skeleton of a pig from Osea Road Red Hill, Maldon, Essex: Colchester Archaeol Group Bull, v 24, p 11-18.

Peltenburg, E J, 1975, Ayios Epiktitos Vrysi, Cyprus: preliminary results of the 1969 1973 excavations of a Neolithic coastal settlement: Prehist Soc Proc, v 41, p 17-45, pls III-VI.

Rajpitak, W and Seeley, N J, 1979, The bronze bowls from Ban Don Ta Phet, Thailand: an enigma of prehistoric metallurgy: World Archaeol, v 11, p 26-31.

Sayed, Abdel Monem A H, 1977, Discovery of the site of the 12th Dynasty port at Wãdi Gawāsis on the Red Sea shore: Rev Egyptol, v 29, p 140-178, pls 8-16. - 1978, The recently discovered port on the Red Sea shore: Jour Egyptian Archaeol, v 64, p 69-71, pl XI.

1980, Observations on recent discoveries at Wādī Gawāsīs: Jour Egyptian Archaeol, v 66, p 154-157, pl XXI.

Simmons, I G, 1975, The ecological setting of Mesolithic man in the highland zone, in Evans, J G, Limbrey, S, and Cleere, H, eds, The effect of man on the landscapethe highland zone: CBA research rept 11, p 57-63.

Waldren, W H, 1979, A Beaker workshop area in the rock shelter of Son Matge, Mallorca: World Archaeol, v 11, p 43-67, pls 4-8.

1981a, Radiocarbon determination in the Balearic Islands-an inventory 1962-1981: Oxford, Donald Baden-Powell Quaternary Research Centre.

1981b, The settlement complex of Ferrandell-Oleza, Valldemosa, Mallorca Spain: Oxford, Donald Baden-Powell Quaternary Research Centre.

Webster, L, 1978, Medieval Britain in 1977, II, pre-conquest: Medieval Archaeol $\mathrm{v} 22, \mathrm{p} 145$. p 238 p 220. 